\title{
Modelling moderated proportional neutron counters using the Geant4 toolkit and the application to detection of fast neutron burst
}

\author{
A. Tarifeño-Saldivia \\ Comisión Chilena de Energía Nuclear (CCHEN), P.O. Box 188-D, Santiago, Chile \\ Center for Research and Applications in Plasma Physics and Pulsed Power, P4,Chile. \\ Chilean Network for Neutron Physics and Applications (CNNPA), Chile \\ E-mail: atarifeno@cchen.cl, atarisal@gmail.com
}

\section{Francisco Molina}

Comisión Chilena de Energía Nuclear (CCHEN), P.O. Box 188-D, Santiago, Chile

Chilean Network for Neutron Physics and Applications (CNNPA), Chile

E-mail: fmolina@cchen.cl

\section{J. L. Tain}

Instituto de Física Corpuscular, CSIC-Univ. Valencia, Valencia, Spain.

\section{Jordan}

Instituto de Física Corpuscular, CSIC-Univ. Valencia, Valencia, Spain.

\section{Soto}

Comisión Chilena de Energía Nuclear (CCHEN), P.O. Box 188-D, Santiago, Chile

Center for Research and Applications in Plasma Physics and Pulsed Power, P4,Chile.

Chilean Network for Neutron Physics and Applications (CNNPA), Chile

\begin{abstract}
The modelling with the Geant 4 toolkit of moderated ${ }^{3} \mathrm{He}$-filled proportional neutron counters is studied. The energy deposition spectra by the neutron capture products in the gas counter is compared with experimental results. On the other hand, efficiency calculation for polyethylene moderated proportional counters are compared with experimental and MCNPX results.Finally, the application of the Geant4 toolkit to the study of the temporal response of moderated proportional neutron counters to detection of bursts of fast neutrons is presented.
\end{abstract}

X Latin American Symposium on Nuclear Physics and Applications (X LASNPA),

1-6 December 2013

Montevideo, Uruguay 


\section{Introduction}

Geant4 is a toolkit for Monte Carlo simulation of the passage of particles through matter. It is widely used in high energy, nuclear and accelerator physics, as well as in studies in medical and space science [1,2]. In the recent years, the Geant 4 collaboration community has been doing efforts in order to improve the toolkit capabilities for the modelling of hadronic processes, in particular for neutrons at low energies. Thus, benchmarking with experimental results and validated codes, e.g. MCNPX, are important activities.

Moderated ${ }^{3} \mathrm{He}$-filled counter tubes are high efficiency fast neutron detectors. In these counters, a thermal neutron undergoes the reaction

$$
{ }^{3} \mathrm{He}+n_{t h} \rightarrow p+t,
$$

where $Q=764 \mathrm{keV}$, being the reaction products energies $E_{p}=573 \mathrm{keV}$ and $E_{t}=191 \mathrm{keV}$. Because of the walls of the counter, in some cases the energy of the reaction products is not completely deposited inside the counter. It results in a deformation of the energy deposition spectra known as wall effect.

\section{Modelling the energy deposition spectra}

The energy deposition spectra from neutron captures in a paraffin moderated ${ }^{3} \mathrm{He}$-filled counter tube was simulated. The detector is a LND2523 neutron proportional counter (see figure 1) inside a paraffin wax block of geometric dimensions $45 \times 15 \times 15 \mathrm{~cm}^{3}$. The moderator is surrounded by a cadmium sheet. In the experiments, the detector was irradiated by an encapsulated ${ }^{252} \mathrm{Cf}$ radioactive neutron source. The modelling of the energy deposition spectra was done according to the following strategy:

- The proportional counter is modeled as a stainless steel tube filled by a mixture of ${ }^{3} \mathrm{He}$ gas and the quenching gas $\left(\mathrm{CO}_{2}\right)$.

- The counter is divided into two volumes: Active and passive(see figure 1). Detection of events is related to energy deposition inside the active volume by the neutron capture reaction products.

- The pure spectra generated by Geant4 should be convoluted with the response function of the detection system. This function is assumed to be a gaussian distribution. The parameters of the gaussian distribution are fitted from the experimental measurement.

- The neutron source is not simulated directly. Instead, the G4GeneralParticleSource class of Geant4 is used for sampling neutrons with the spectra of a $252 \mathrm{Cf}$ radioactive neutron source (ISO8529-1)[3]. The neutrons are generated isotropically from a region with same geometry of the neutron source.

- The simulation use the QGSP_BIC_HP PhysicsList for the physical processes. The QGSP_BIC_HP is a recommended PhysicsList for neutrons in shielding, low energy dosimetry, medical and industrial applications [4]. 


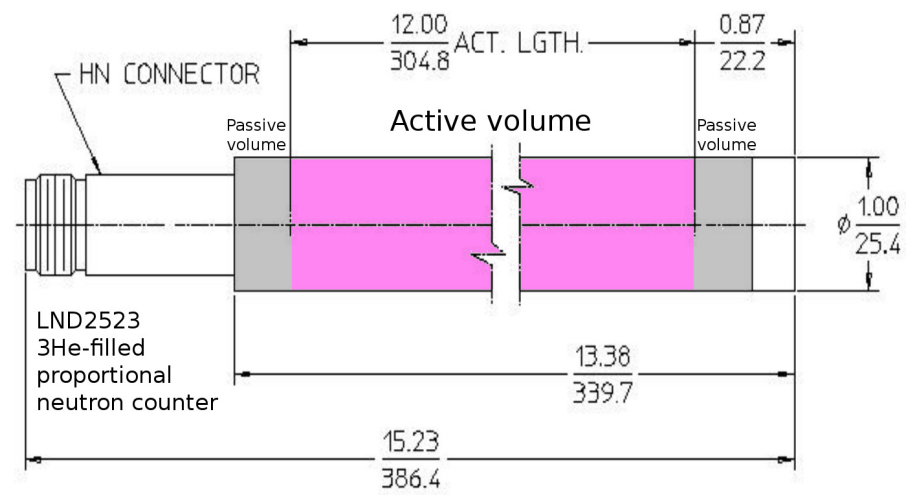

Figure 1: Diagram of the LND2523 proportional counter. Dimensions in inches and milimeters for upper and lower number respectively.

\subsection{Results and remarks:}

In figure 2 are shown the results of the Geant 4 simulation of the energy deposition spectra. A good agreement is obtained beetwen the simulated and the experimental spectra. In contrast, poor agreement is obtained when comparing the calculated to the measured detector eficiency. From simulations is concluded that:

- To overcome the problems with the calculated efficiency, a special treatment of neutron scattering bellow $5 \mathrm{eV}$ is required. This treatment is provided by the G4neutronHP package [5]. For this treatment, the molecular structure or crystal lattice plays an important role, being neccesary a special thermal library for each material. At present, there is not such library for paraffin wax.

- Parameters such as: Tube gas pressure, quenching gas proportion, geometry of the neutron source, density and paraffin composition do not have a significative influence in the shape of the energy deposition spectra.

- On the other hand, the energy of the moderated neutrons and their entering direction to the counter affect the shape (height and slope) of the part of the spectra to the left of the full energy deposition peak.

- The effects on the shape of the spectra are due to the existence of the interface active/passive volume. The effect of the energy of the moderated neutrons is related to the mean free path of these neutrons inside the counter. While, the effect of the entering direction is related to the capture of the neutrons in the active or passive volume.

- The effects on the shape of the deposition energy spectrum by the capture reaction products in ${ }^{3} \mathrm{He}$-filled proportional counters may be of interest for slow neutron spectroscopy. 


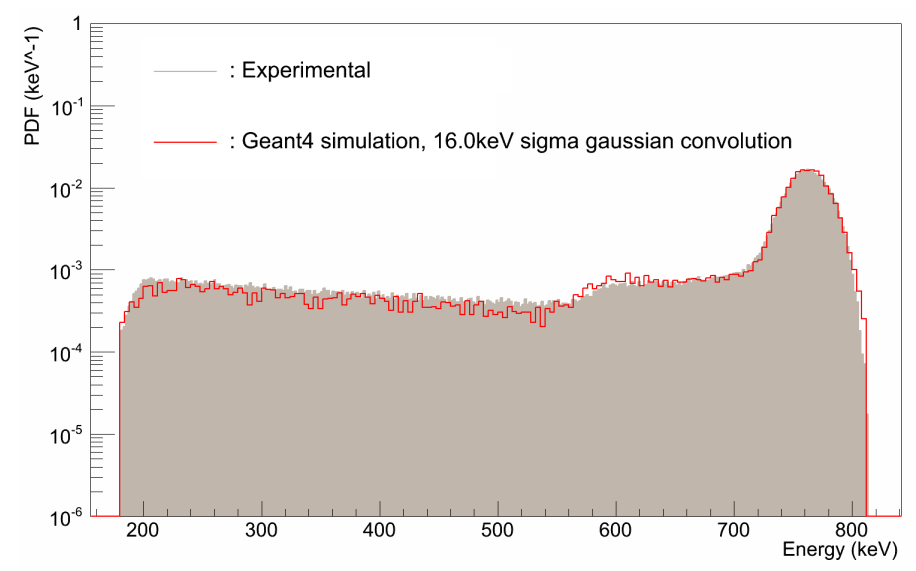

Figure 2: Results of the modelling of the energy deposition spectra.

\begin{tabular}{cc}
\hline $\begin{array}{c}\text { Detector } \\
\text { number }\end{array}$ & $\begin{array}{c}\text { Polyethylene block } \\
\text { size }\left(\mathrm{cm}^{3}\right)\end{array}$ \\
\hline 1 & $4.5 \times 4.5 \times 70.0$ \\
2 & $7.0 \times 7.0 \times 70.0$ \\
3 & $12.0 \times 12.0 \times 70.0$ \\
4 & $18.0 \times 18.0 \times 70.0$ \\
5 & $22.5 \times 22.5 \times 70.0$ \\
6 & $27.0 \times 27.0 \times 70.0$ \\
\hline
\end{tabular}

Table 1: Moderator geometries for experiments and calculations of total efficiency.

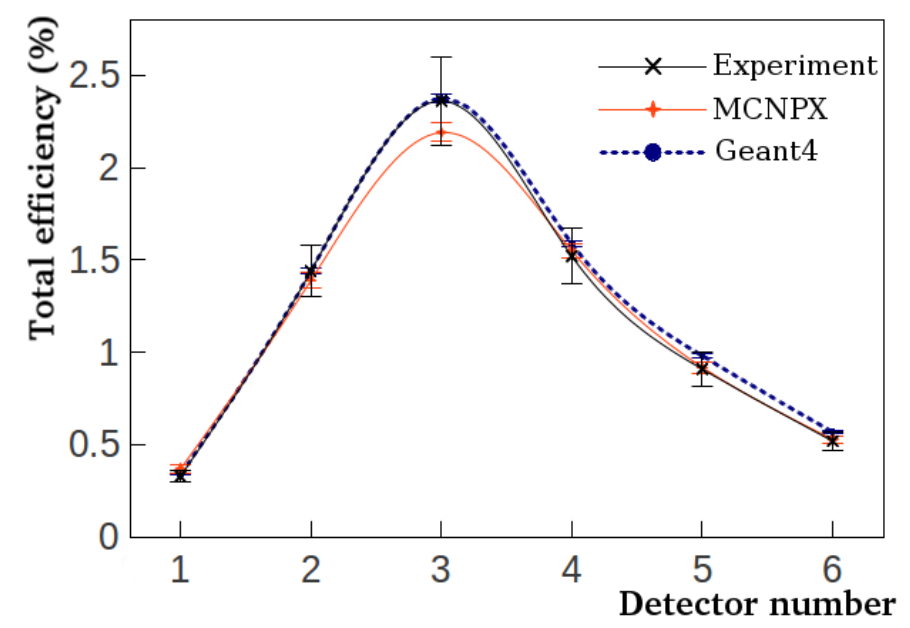

Figure 3: Comparison of calculated with experimental efficiencies. 


\section{Efficiency calculations for polyethylene moderated counters}

The total efficiency of ${ }^{3} \mathrm{He}$-filled counter tubes moderated by polyethylene blocks of different size was calculated. The calculations are compared with experimental measurements and MCNPX calculations by Jordan et. al. [6]. The detectors are six polyethylene moderated LND252248 counters tubes. The moderator geometries are given in table 1. Each detector was irradiated by a ${ }^{252} \mathrm{Cf}$ radioactive neutron source. The modelling of the total efficiency was done according to the following strategy:

- The counter is modeled using active and passive volumes.

- The use of thermal libraries is included in the PhysicsList and the thermal library for polyethylene ("TS_H_of_Polyethylene") is used.

- For sampling the neutron energy from the source, the Watt fission spectrum $(f(E)=C$. $\left.e^{-E / a} \cdot \sinh (\sqrt{b E})\right)$ was used with the MCNP recommended parameters for a ${ }^{252} \mathrm{Cf}$ neutron source $\left(a=1.025 \mathrm{MeV}\right.$ and $\left.b=2.926 \mathrm{MeV}^{-1}\right)$.

\subsection{Results and remarks}

In figure 3 is shown the comparison beetwen Geant 4 calculations with experimental measurements and MCNPX calculations. Within the experimental uncertainties of the measurements, good agreement is obtained with both numerical calculations (Geant4 and MCNPX). When comparing Geant4 with MCNPX calculations, a systematic bias is observed for Geant4 results.

- The systematic bias is related to some bugs arising from the way Geant4 extrapolate data from libraries[7]. These bugs are expected to be corrected in the next releases of Geant 4.

- For efficiency calculations, the physical description of the neutron source plays an important role. In addition to the use of the proper spectra for sampling neutrons, the proper size and geometry of the source should be used in order to obtain good results.

\section{Application to detection of fast neutron burst}

Fast neutron burst are produced in fusion plasma experiments, e.g. plasma focus, Z-pinch or laser driven inertial confinement. In these experiments, fusion reactions happen during short time periods, typically less than 500ns. For a neutron burst, the piling-up of single events detected by a moderated proportional neutron counter produce an only signal. This signal can be recorded by an osciloscope and used for the measurement of the neutron burst intensity (neutron yield) [8]. The shape of the signal depends on the feeding time of moderated neutrons to the counter and the response of the adquisition system (preamplifier). The reconstruction of the signal is modeled using Geant4. Geant4 is able to track one independent event and its secondaries. Therefore, the direct simulation of a neutron burst is not possible. The reconstruction of the detector response is done using the following strategy: 

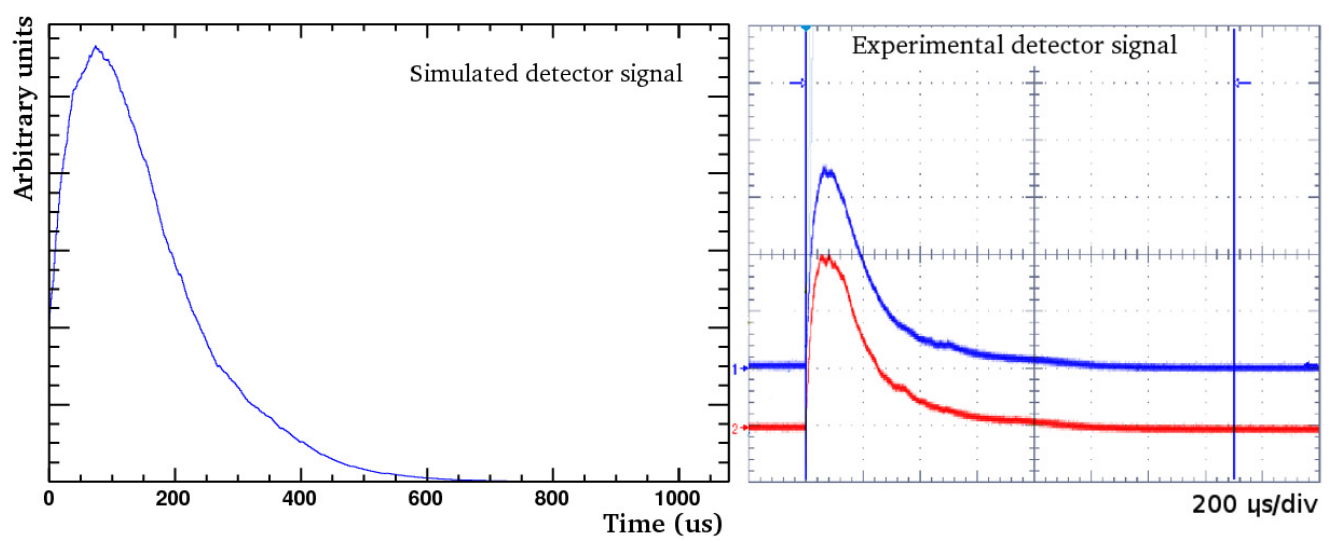

Figure 4: Comparison of a Geant4 simulated signal with experimental measurements by two paraffin moderated ${ }^{3} \mathrm{He}$-filled counters for a neutron burst of $10^{6} n /$ burst .

- The neutron events travelling inside the moderator and detected by the counter are assumed to be independent to each other.

- The temporal response of the adquisition system, measured experimentally, and the Geant4 calculated passage time of neutrons through the moderator before being detected is used for reconstruction of the signal.

\subsection{Results and remarks}

In figure 4 is shown a comparison of Geant 4 simulated signal with experimental measurements for a neutron burst of $10^{6} n$ /burst. These preliminary result shows a similar shape of the simulated and the measured signals. The numerical reconstruction of recorded signal may be of interest to study loss of proportionality in moderated proportional counters when irradiated by neutron bursts.

\section{Concluding remarks and future work}

The use of the Geant4 toolkit (geant4.V9.5) is showing promising results for the modelling of moderated ${ }^{3} \mathrm{He}$-filled proportional neutron counters. Future work is related to the validation of a modelling code able to calculate properly the energy deposition spectrum by the neutron capture reaction products and the detector total efficiency. For this purpose, a measurement campaign is underway to provide reliable data for benchmarking and validation.

\section{Acknowledgments}

Research by Thermonuclear Plasma Department from CCHEN is supported by Conicyt grant ACT-1115.

\section{References}

[1] S. Agostinelli et. al., Nuclear Instruments and Methods in Physics Research A, 506, 250-303, 2003. 
[2] J. Allison et. al., IEEE Transactions on Nuclear Science, 53(1), 270-278, 2006.

[3] ISO 8529-1. Reference neutron radiations - Part 1: Characteristics and methods of production

[4] geant4.cern.ch/support/proc_mod_catalog/physics_lists/useCases.shtml

[5] wwwae.ciemat.es/ arce/particle_hp/BriefInstructions_G4NeutronHP_v5. $\operatorname{pdf}$

[6] D. Jordan et. al., Astroparticle Physics , 42, 1-6, 2013.

[7] "Validation of the thermal neutron physics in GEANT4" A. R. García, E. Mendoza and D. Cano-Ott. G4 Hadronic group meeting, 17th of April 2013.

[8] "Calibration methodology for proportional counters applied to yield measurements of a neutron burst" A. Tarifeño-Saldivia et. al., Rev. Sci Instrum., in press. 